A.J. Morales ${ }^{1}$, Jonathan Reyes ${ }^{1}$, Peter H. Joo ${ }^{1}$, Isaac Boxx ${ }^{2}$, Kareem A. Ahmed ${ }^{1}$ Pressure Gradient Tailoring Effects on the Mechanisms of Bluff-Body Flame Extinction

Combustion and Flame, 215 (2020) 224-237

${ }^{1}$ Center for Advanced Turbomachinery \& Energy Research, Department of Mechanical and Aerospace Engineering, University of Central Florida, Orlando, USA

${ }^{2}$ Institute for Combustion Technology, German Aerospace Center

The original publication is available at www.elsevier.com

https://doi.org/10.1016/j.combustflame.2020.01.035

(C) $\langle 2020\rangle$. This manuscript version is made available under the CC-BY-NC-ND 4.0 license http://creativecommons.org/licenses/by-nc-nd/4.0/ 


\section{Pressure Gradient Tailoring Effects on the Mechanisms of Bluff-Body Flame Extinction}

Anthony J. Morales ${ }^{\text {a }}$, Jonathan Reyes ${ }^{\text {a }}$, Peter H. Joo ${ }^{\text {a }}$, Isaac Boxx ${ }^{b}$, Kareem A. Ahmed ${ }^{\text {a }}$

${ }^{a}$ Center for Advanced Turbomachinery \& Energy Research, Department of Mechanical and Aerospace Engineering, University of Central Florida, Orlando, Florida, 32816, USA

${ }^{\mathrm{b}}$ Institute of Combustion Technology of the German Aerospace Center, Deutsches Zentrum für Luft- und Raumfahrt (DLR), Institut für Verbrennungstechnik, Stuttgart, Germany

Full-length article

Keywords:

Turbulent premixed combustion

Bluff-body Flame blowout

Strain rate

Vorticity mechanisms

\section{Abstract}

The mechanisms of flame blowout under pressure gradient effects are explored for a bluff-body stabilized flame. The blowout process is induced through equivalence ratio reduction from a lean stabilized flame to complete blowout. Simultaneous high-speed particle image velocimetry (PIV) and $\mathrm{C}_{2}{ }^{*} / \mathrm{CH}^{*}$ chemiluminescence imaging diagnostics are used to obtain the instantaneous flame structure, vorticity field, equivalence ratio, and local strain rate during the extinction process. The goal is to elucidate the effect of flame-generated vorticity on lean flame extinction. Three test-sections configured as a nozzle, a rectangular duct, and a diffuser, are used to alter the downstream pressure gradient yielding high, nominal, and low magnitudes of flamegenerated baroclinic torque. For all three configurations, the flame brush narrows and the shear layer vorticity expands in the transverse direction resulting in flame-shear interactions and extinction. The flame-shear layer interaction increases the strain rate along the flame; however, the strong flame-generated vorticity for the nozzle case delayed the strain rate increase by keeping the flame away from the shear layer the longest. The sharp increase in the Karlovitz number above unity caused by the sudden increase in the strain rate corresponds to the time of flame brush contraction and shear layer width expansion. It is shown that the downstream pressure gradient can either augment or attenuate the time required for the Karlovitz number to reach a critical value of unity, which is associated with local extinctions along the flame. In all of the test-section configurations, the flame-generated vorticity has a weak influence on the Bénard von Kármán (BVK) instability mode and its harmonics. The Strouhal number during blow-out 
remained relatively constant in all of the cases showing greater sensitivity to the shear layer length than to the BVK frequency.

\section{Introduction}

Flame stability is a persistent concern within propulsion and energy technologies, such as ramjet engines and gas turbines, where combustion systems are used to convert stored chemical energy into propulsive and electrical power [1,2]. The flame stabilization process occurs over limited conditions, and in the effort to mitigate pollutant emissions such as NOx and $\mathrm{CO}$, these combustion devices operate close to the lean limit [3]. However, operating near the lean combustion limit increases the susceptibility of flame blowout in high-speed flows when disturbances, such as a sudden reduction of fuel flow, occur in the system [4-6]. Hence, a better understanding of flame stabilization in high-speed flows is needed to further the development of turbulent combustion technologies and advanced engines.

A common method of flame stabilization in high-speed flows is through using a bluff-body that is placed in the flow path. The bluff-body induces flow separation, forming a recirculation zone within the immediate bluff-body wake $[7,8]$. The recirculation zone provides long residence times for flames to anchor and stabilize through turbulent flow interactions between freestream cold reactants and recirculating combustion products [7-10]. The operational range of bluff-body flames, however, are bounded by stability performance limits that are a function of inflow velocity, fuel-air ratio, temperature, pressure, and bluff-body geometry [11-14]. Outside of these bounds, the flame is subjected to excessive fluid dynamic and hydrodynamic strain and results in a global flame extinction and blow-out $[5,6,11,12]$.

Considerable attention has been garnered for bluff-body stabilized turbulent premixed flames over many decades [15-17] to provide a thorough understanding of the broad features of flame anchoring and extinction. For instance, Nair and Lieuwen have documented global flame oscillations of bluff-body flames near blowout [5]; this oscillatory behavior was found to be reminiscent of the Bénard von Kármán (BVK) vortex shedding instability. This global instability was hypothesized to promote high flame curvatures while imparting an oscillatory hydrodynamic strain rate upon the flame boundary [4-6]. Increased curvature and hydrodynamic straining both contribute to overall flame stretch and cause localized extinctions along the flame [5]. Chowdhury et al. [18] found that the strain rate magnitudes and frequency of localized 
extinctions intensify as the equivalence ratio is reduced. This discussion was augmented by Tuttle et al. [19], who resolved the local strain rate along a bluff-body flame and determined that the spatial locations where the flame experiences the largest strain rates correspond to regions where the flame boundary overlaps with the convecting Kelvin-Helmholtz shear layer vorticity. Additionally, Chaudhuri et al. [20] concluded that as the equivalence ratio is reduced, the laminar flame speed will decrease and allow the flame to collimate and interact with the shear layer vorticity. The flame-shear layer interaction drives high strain rates along the flame and results in localized extinction events that consequently lead to global blowout. The culmination of these research studies have deduced that flame-vortex interactions are a key driving mechanism of lean blowout. However, additional details of the temporal dynamics of the extinguishing flame-flow field are still desired to fully characterize and validate these mechanisms.

To further characterize the extinguishing vorticity dyanmics within the flow field, it is beneficial to study the combustion-induced vortex mechanisms of dilatation and baroclinic torque from the vorticity transport equation. These two vorticity mechanisms are generated by the presence of the flame within the flow field and act to stabilize the flame [21]. The dilatation vorticity mechanism is directly dependent upon the density ratio between the reactants and products, and is associated with the gas expansion of the combustion products within the flow domain [22,23]. Dilatation also acts as a vortex sink within the reacting flow domain aiding in flame stabilization through suppression of the BVK vortex shedding instability [6,22]. The baroclinic torque mechanism is expressed as $1 / \rho^{2}(\nabla \rho \times \nabla P)$ and is produced from the misalignment of the axial pressure gradient, $\nabla P$, and the density gradient, $\nabla \rho$, between unburnt reactants and burnt products across the thickness of the flame [6,21,22,24]. Baroclinic torque is unique from other vorticity mechanisms as it generates new vorticity along the boundary of the flame and has the most significant influence on the flame structure, velocity fields, turbulence, and heat release when compared to the other vortex mechanisms [6,24,25]. These combustioninduced vortex mechanisms play a crucial role for achieving flame stabilization; however, when operating at low equivalence ratios, the magnitudes of dilatation and baroclinic torque are reduced, leading to flame structural instabilities and exacerbated flame stretching [5,6,18-20]. More specifically, the vorticity generated from the baroclinic torque mechanism acts in the opposite direction relative to the shear layer vorticity within the recirculation zone [26]; in this 
manner, the superposition of the baroclinic torque attenuates the shear layer vorticity (or shear layer roll-up) monotonically along the axial distance $[6,26]$.

Recent studies from Morales et al. [27] have experimentally characterized the temporal mechanisms of bluff-body flame extinction. The results showed the role of baroclinic torque during flame blowout and its effects on flame-vortex interaction dynamics. It was demonstrated that the temporal reduction of baroclinic torque allows for the magnitude of the shear layer vorticity to increase. Simultaneously, the flame boundary collimates and is pushed toward the shear region, exposing the flame to high vorticity magnitudes. The increased vorticity magnitudes experienced by the flame were shown to correlate to the increase in strain rate along the flame. In other words, the flame-vorticity interaction dynamics expose the flame boundary to high strain rate magnitudes that result in localized extinctions and flame blowout.

The current research seeks to build upon recent investigations of flame blowout and highlight the role of baroclinic torque throughout the extinction process. To do so, lean blowout is investigated within a bluff-body combustor coupled with high-speed optical diagnostics. The role of flame-generated baroclinic torque on the extinction process is explored by changing the magnitude of baroclinic torque through tailoring the axial pressure gradient within the combustor using nozzle, rectangular, and diffuser configurations. The goal of the current work is to provide clarity and additional insights into the role of baroclinicity on the flame-votex interaction dynamics that cause extinction. In doing so, this work also validates the temporal blowout mechanisms previously hypothesized. 


\section{Experimental Methodology}

\subsection{Combustion Facility}

Experiments were conducted in a high-speed combustion facility. The combustion facility is an enclosed flow channel that is designed for high-speed flows with a bluff-body and an optically accessible test section. The schematic of the facility is provided in Fig. 1. The facility consists of a conical diffuser with an inlet diameter of $50 \mathrm{~mm}$ and a rectangular exit with an internal height and width of 157 and $127 \mathrm{~mm}$, respectively. The rectangular plenum has an overall length of $2.250 \mathrm{~m}$. Square mesh screens are placed between the plenums for flow conditioning. The mesh screens have a wire diameter of $1 \mathrm{~mm}$ and a characteristic length of $4 \mathrm{~mm}$ that yields an open area of $64 \%$. A fifth-order polynomial nozzle reducer is attached immediately following the plenum and accelerates the flow to the bluff-body. A ballistic-type bluff-body was used to minimize the flow separation at the leading edge and as a point of flame anchoring at the trailing edge. The height and length of the bluff-body are 16 and $64 \mathrm{~mm}$, respectively, providing a blockage ratio of $\sim 30 \%$. This design is advantageous when compaired to other bluff-body geometries: it reduces the drag over the bluff-body, increases the range of inflow velocities in which flames can be stabilized, and extends the weak extinction limits to lower equivalence ratios [13].

The bluff-body is positioned on the axial centerline and spans across the full width of the test section. A schematic depicting the bluff-body is provided in Fig. $1 b$. Immediately following the bluff-body is an optically accessible test-section. The height and width of the test-section are 45 and $127 \mathrm{~mm}$, respectively. The test-section can be configured as a diffuser, a nozzle, or as a

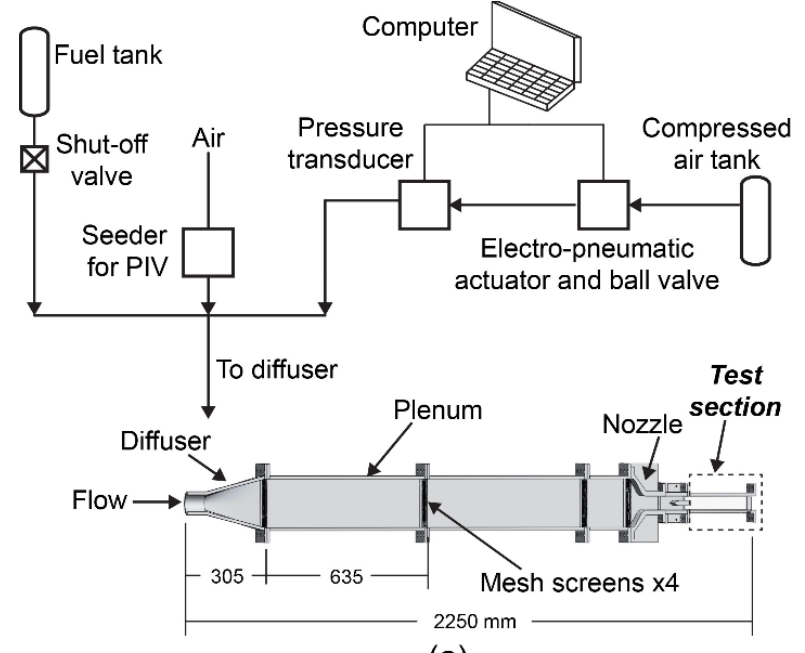

(a)

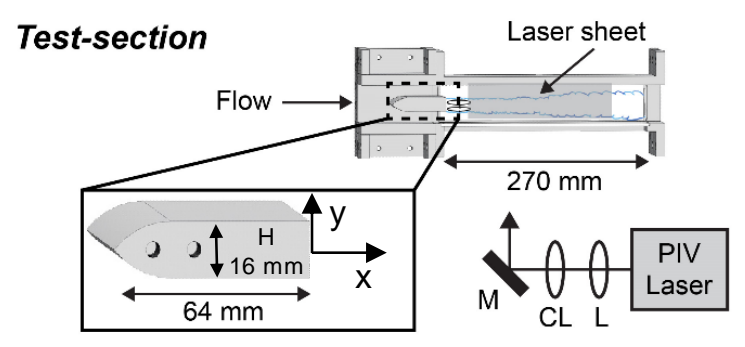

(b)

Figure 1. Schematic of experimental facility. a) Blow down combustion facility and b) bluff-body flame stabilizer, coordinate axes, and optically accessible test section. 
straight rectangular duct (also known as the nominal configuration), as presented within Fig. 2. The diffuser and nozzle configurations are achieved via 3-degree angled wedges that adjust the orientation of the upper and lower walls of the test section.

Three of the four test section walls are recessed and fitted with fused quartz silica glass to allow for optical diagnostics. The upper surface of the test section is the only one that is not optically accessible, and is also painted black to minimize light scattering. Static pressure ports are also installed along the axial length of the upper wall to quantify the axial pressure profiles for each of the
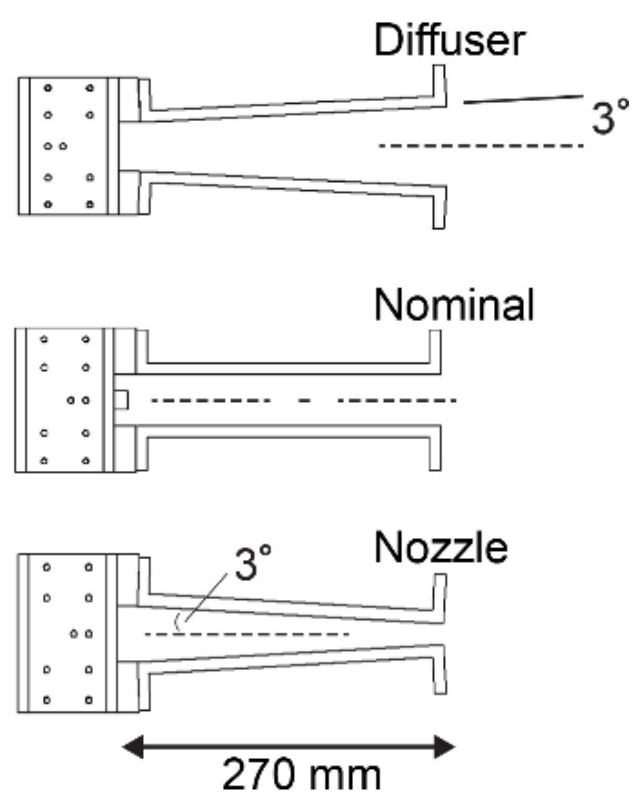

Figure 2. Test-section configurations showing the diffuser, nominal, and nozzle geometries. The various geometries are achieved from 3-degree angles wedges. configurations within Fig. 2. The pressure ports have an inner tube diameter of $1 \mathrm{~mm}$ and are mounted flush with the inside wall. The pressure taps are evenly spaced every $3.18 \mathrm{~mm}$ along the length of the upper wall to provide an adequate spatial resolution to characterize the axial pressure profiles within the test-section. The pressure ports are connected through small plastic tubes to a Scanivalve system with PDCR-23d pressure transducers. Pressure measurements are acquired at a sampling frequency of $2500 \mathrm{~Hz}$ with 3000 total samples collected. The uncertainty of the pressure measurements is nominally $\pm 8 \mathrm{~Pa}$, and the corresponding uncertainty in the axial pressure gradient is $\pm 0.46 \mathrm{~Pa} / \mathrm{m}$. The mean pressure at each downstream location is used to quantify the axial pressure profile and pressure gradient within the test section for all three geometry configurations presented within Fig. 2.

\subsection{Flow control system}

Air flow was regulated using an electro-pneumatic valve (JFLow Series DM4500) upstream of the experimental facility. The valve was controlled in a feedback loop using a pressure transducer (Dwyer Series 626) and a proportional-integral (PI) controller. A computer program controlled the PI controller and adjusted the pneumatic valve position to obtain the desired air flow velocity within the optical viewing region. To obtain the desired equivalence ratio of $\Phi=$ 
0.7, gaseous propane was injected into the bulk air flow upstream of the flow conditioning plenum. The propane flow rate was controlled with a rotameter, regulator, and a soleniod valve. The free-stream velocity of the propane-air mixture within the test-section was maintained at 25 $\mathrm{m} / \mathrm{s}$ along the trailing edge of the bluff-body for all test conditions. Spark-wire ignited the combustible mixture and flame extinction was achieved by rapidly stopping the propane flow rate using the soleniod valve. Simultaneous high-speed PIV and chemiluminescence diagnostic techniques recorded the transient flame blow-out process.

\subsection{Optical Diagnostics}

Three high-speed imaging systems were employed simultaneously in this study: 1) a widefield, two-component particle image velocimetry (PIV) system, 2) a narrow-field, high spatial resolution stereoscopic PIV (SPIV) system, and 3) a $\mathrm{C}_{2}{ }^{*} / \mathrm{CH}^{*}$ chemiluminescence imaging system [27,28]. The utility of having two overlapping PIV systems is that it enables one to put high-resolution, narrow-field SPIV measurements into the context of the overall flow-field. Each system is described below and the general schematic of the optical setup is provided within Fig. 3.

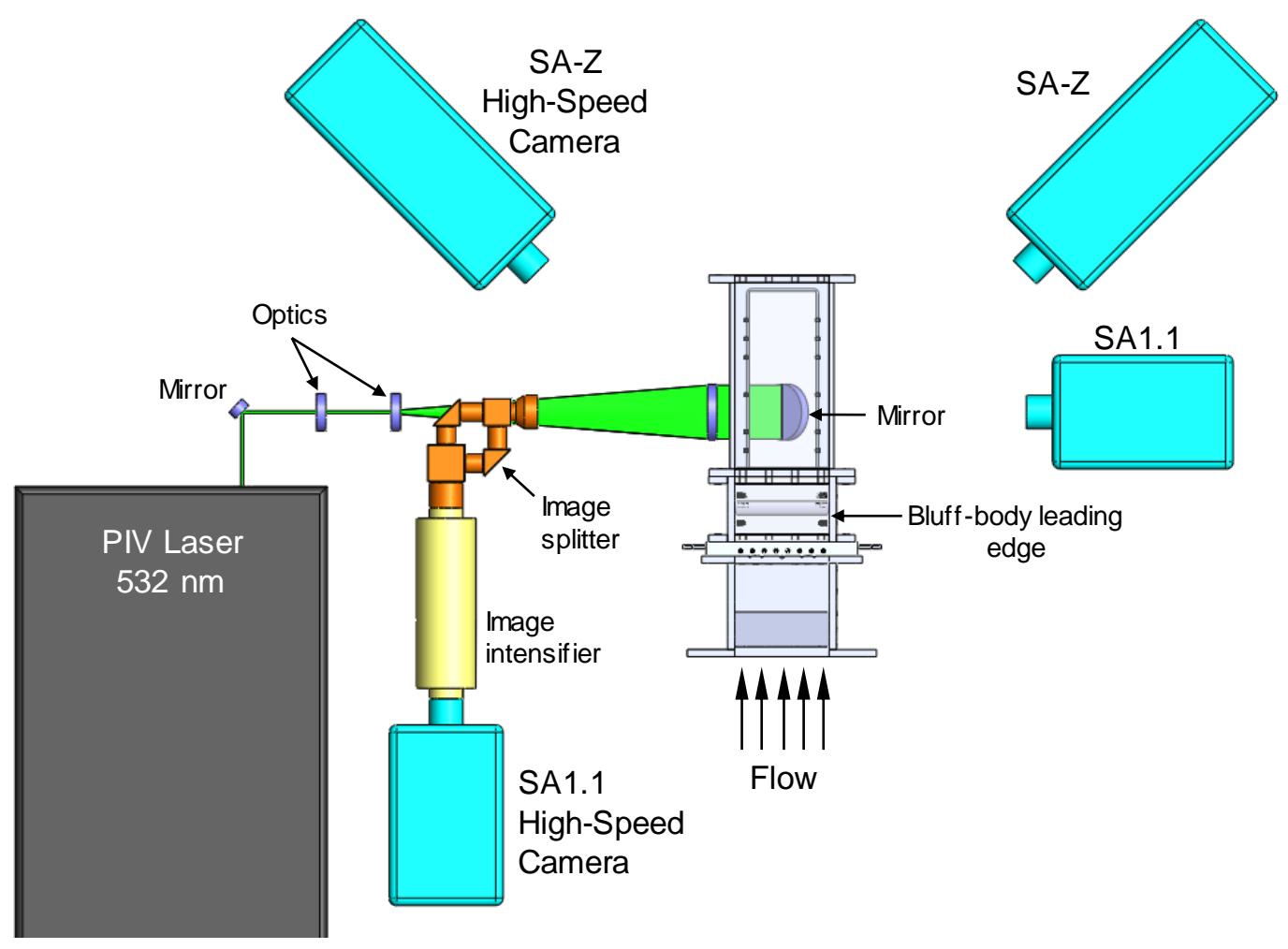

Figure 3. Top-down view of the test section and optical setup. 


\subsubsection{Wide-Field PIV}

The wide-field PIV system consists of a high-speed laser, sheet-forming optics, and a camera mounted perpendicularly to the flow facility; this is depicted within Fig. 3. A dual-cavity, diode pumped solid state $\mathrm{Nd}$ :YAG laser is used to illuminate aluminum oxide $\left(\mathrm{Al}_{2} \mathrm{O}_{3}\right)$ seed particles within the flow. The seed particles have a nominal diameter of $150 \mathrm{~nm}$ and are injected into the main bulk flow via a pressure-driven swirl seeder. Once pressurized, the particles mix within the seeder and an in-line ball value is opened to allow the seed to enter the reactants upstream of the flow conditioning plenum. To prevent the seed particles from agglomerating, the seeder also inclides two mesh screens, each with a mesh size of $20 \mu \mathrm{m}$. The seed and reactants flow through the plenum to ensure a premixed mixture of uniform seed density reaches the bluff-body combustor. To illuminate the particles within the combustor, a $532 \mathrm{~nm}$ dual head laser is operated sequentially to produce $12 \mathrm{~mJ}$ pulses with $20 \mathrm{kHz}$ repetition rate; the time separation for each pulse pair is $50 \mu \mathrm{s}$. The laser beam is formed into a sheet using a pair of cylindrical lenses (focal lengths $-25 \mathrm{~mm}$ and $+450 \mathrm{~mm}$ ) and directed into the test section using a $1000 \mathrm{~mm}$ focal length cylindrical lens and a mirror mounted below the test section.

Elastic scattering from the alumina particles was imaged on a high-speed Photron SA1.1 camera mounted perpendicular to the flow facility, as shown within Fig. 3. The camera is equipped with a $50 \mathrm{~mm}$ focal length, $f / 1.2$ imaging lens and collects images over an array comprised of $1024 \times 1024$ set to read out in partial frame mode $512 \times 379$ pixels across a field of view of $61 \times 45 \mathrm{~mm}$. The wide-field spatial domain encompasses the bluff-body recirculation zone and reattachment region between $0.55 \leq x / H \leq 4.75$. The corresponding spatial resolution of the wide-field PIV images is $125 \mu \mathrm{m} /$ pixel. The images are processed within PIVLab 1.35 software [29] and validated using LaVision Davis software. The images are first preprocessed with a contrast limited adaptive histogram equalization (CLAHE) filter to enhance the contrast of the images [30]. Velocity vector fields were determined via a four-step multi-pass crosscorrelation algorithm beginning with an initial interrogation region of $64 \times 64$ pixels and reducing to a final grid of $16 \times 16$ pixels with a $50 \%$ overlap for each interrogation step. The final vector spacing is $\lambda_{m}=950 \mu \mathrm{m}$, which is $\lambda_{m} l_{f}=2$ relative to the laminar flame thickness of a propane-air flame at $\Phi=0.7$, and $\lambda_{m} / \lambda_{k}=14.1$ relative to the Kolmogorov length scale. The statistical correlation uncertainty in the velocity is $0.25 \mathrm{~m} / \mathrm{s}$ [31]. 


\subsubsection{High-Resolution Stereoscopic PIV}

The SPIV system consisted of a high-speed laser and a pair of high-speed Photron SA-Z cameras mounted on opposite sides of the facility, as shown in Fig. 3. The SPIV cameras were equipped with a Tamron $180 \mathrm{~mm}$ focal length $f / 4$ macro lens as well as scheimpflug adaptors to mitigate the image blur and adjust the plane of focus to align with the laser sheet. The field of view for the SPIV system is nested within the wide-field system, allowing for a single laser (the $532 \mathrm{~nm}$ ND:Yag laser described in Section 2.3.1) to illuminate the flow field for both diagniostics. This also ensures that images are collected simultaneously across both diagnostics at the same frequency of $20 \mathrm{kHz}$. The SPIV cameras are focused on the region between $1.8 \leq x / H$ $\leq 3.4$ to highlight the area where global blowout is expected to initiate. This region covers a spatial domain of $24 \times 24 \mathrm{~mm}$ and images are collected over a sensor comprised of $1024 \times 1024$ pixels. This results in a spatial resolution of $24 \mu \mathrm{m} /$ pix for the SPIV images. The SPIV images are processed in the same manner as the wide-field images utilizing a four-step multi-pass method, however, the initial window size was $200 \times 200$ pixels and reduced to a final window size of $25 \times 25$ pixels with a $50 \%$ overlap. The SPIV velocity vector spacing is $\lambda_{m}=292 \mu \mathrm{m}$, corresponding to $\lambda_{m} / l_{f}=0.89$, and $\lambda_{m} / \lambda_{k}=4.1$. The statistical correlation uncertainty in the velocity fields from the SPIV images is $0.05 \mathrm{~m} / \mathrm{s}$.

\subsection{3 $\mathrm{C}_{2}{ }^{*} / \mathrm{CH}^{*}$ Chemiluminescence Imaging}

The ratio of the diatomic carbon $\left(\mathrm{C}_{2}{ }^{*}\right)$ and methylidene $\left(\mathrm{CH}^{*}\right)$ radical intensities have been shown to index the equivalence ratio of the reaction mixture [27,28]. The production of these radicals is dependent on the fuel and air mixture composition during the reaction and correlate to the local equivalence ratio. The $\mathrm{C}_{2}{ }^{*} / \mathrm{CH}^{*}$ chemiluminescence imaging system was used to determine the equivalence ratio throughout the duration of flame extinction. The setup consisted of an imaging objective, an image splitter, two band-pass filters, intensifier, and a high-speed SA1.1 camera, as depicted within Fig. 3. Self-excited chemiluminescence from the $\mathrm{C}_{2}{ }^{*}$ and $\mathrm{CH}^{*}$ flame radicals are collected through a $50 \mathrm{~mm}$ Nikon Nikkor, f/1.2 objective lens mounted to an image splitter. Narrow-band wavelength filters are used to isolate the $422-432 \mathrm{~nm}$ band of $\mathrm{CH}^{*}$ and 505-522 nm band of $\mathrm{C}^{*}$ chemiluminescence, and the filtered signals are imaged onto the camera with the aid of an external lens-coupled intensifier. The configuration resulted in a field of view of $61 \times 45 \mathrm{~mm}$. The method to determine the equivalence ratio of the reaction has been discussed by Reyes et al. [28] and only a brief description is provided here. Firstly, background 
image noise level that is induced from the intensifier is subtracted from the $\mathrm{C}_{2}{ }^{*}$ and $\mathrm{CH}^{*}$ images; the background noise level is selected to ensure that the equivalence ratio determined from this technique matches the experimentally set equivalence ratio prior to flame blowout $(\Phi=0.7)$. All intensity values below this determined background noise level are removed from both $\mathrm{C}_{2}{ }^{*}$ and $\mathrm{CH}^{*}$ images and the ratio of $\mathrm{C}_{2}{ }^{*} / \mathrm{CH}^{*}$ is evaluated. The ratio of $\mathrm{C}_{2}{ }^{*} / \mathrm{CH}^{*}$ correlates to the equivalence ratio, as demonstrated by Reyes et al [28]. To capture lean equivalence ratios for $\Phi$ $<0.7$, calibration images are collected for stable reacting propane-air flames using the same setup and optics as the extinction experiment. Stable lean equivalence ratios ranging from $0.5 \leq$ $\Phi \leq 0.8$ with incremental steps of 0.05 are maintained and a total of $1000 \mathrm{C}_{2}{ }^{*}$ and $\mathrm{CH}^{*}$ images are collected for each equivalence ratio. The average $\mathrm{C}_{2}{ }^{*} / \mathrm{CH}^{*}$ intensity ratio is calculated for each $\Phi$ to develop a calibration curve. The calibration curve is used to determine the equivalence ratios through the duration of the extinction process. This method is advantageous, as it allows for variations in $\Phi$ to be determined locally within the domain as the extinction process occurs. The equivalence ratio data will be presented and discussed in the results section below.

\subsection{Flame Edge Detection}

The instantaneous local position of the flame is determined from the seed particle density step change from the wide-field PIV images and validated with both the $\mathrm{C}_{2}{ }^{*}, \mathrm{CH}^{*}$ and SPIV images. The PIV seed-particle density jump identifies the interface between the reactants and products from the high-heat release along the flame and has been implemented to yield reliable flame contours for turbulent flames at moderate Reynolds numbers [19,32-34].

The wide-field flame boundary is obtained by first accentuating the seed particle density jump interface by applying three passes of a median filter within a $3 \times 3$ pixel window size. To

enhance the contrast between the products and reactants within the flow, the complement of the median filtered image is determined. The flame boundary is determined by a Moore-Neighbor tracing algorithm, which identifies the boundary of the flame using a specified threshold [35]; the specific threshold value for each image is determined from Otsu's method [36] which separates the image into two clusters of pixels: one cluster highlights the flame topology and the other consist of the dark background. The appropriate threshold is calculated as the value which minimizes the variance within the each cluster, or equivalently, maximizes the variance between 
the two clusters. This method allows for the instantaneous flame coordinates to be extracted throughout the duration of the extinction process.

The same flame trace method was applied to the stereo PIV images, to validate the position of the flame. The root mean square (RMS) difference of the flame position between the two stereo cameras was negligible throughout the entire extinction process with a difference of less than $5 \%$ of a bluff-body height which is approximately on the order of the wide-field PIV vector spacing $\sim 0.8 \mathrm{~mm}$. The RMS difference between the stereo images and the wide-field PIV images was between 10-15\% of a bluff-body height throughout the extinction process. The wide-field PIV flame coordinates were also validated with the trace of the flame images obtained from the $\mathrm{C}_{2}{ }^{*} / \mathrm{CH}^{*}$ imaging diagnostics, which specifically captures the chemiluminescence of the reacting regions. The $\mathrm{C}_{2}{ }^{*} / \mathrm{CH}^{*}$ flame boundary also maintained an RMS difference of less than $15 \%$ of a bluff-body height throughout the duration of extinction.

\subsection{Flame Blowout Methodology}

Premixed propane and air enter the combustion test section at a temperature of $300 \mathrm{~K}$ and an equivalence ratio of $\varphi=0.7$. The mixture is ignited, and a stable lean flame is maintained for a short duration ( 2-4 seconds) to minimize any effects of preheating the bluff-body. Once a lean flame is stabilized, the fuel flow rate into the reactant mixture is shutoff via a solenoid valve to induce global blowout. The response time of the solenoid valve is $0.1 \mathrm{~s}$, which is 2000 times longer than the data sampling time $(0.05 \mathrm{~ms})$ and $\sim 6-100$ times longer than the chemical $\left(\tau_{E S R}\right)$ and fluid time scales for the flame-flow field [6]. This ensures that the relevant physcis can be temporally captured using the diagnostics listed in Section 2.3. After each blowout event, room temperature air continues to flow over the bluff-body to bring the temperature back to $300 \mathrm{~K}$ prior to re-ignition. Similar methods have also been implemented in recent experiemtnal and numerical work $[27,41]$, and have been shown to capture the relevant flame-flow physcis.

The pixel intensity of the chemiluminescence images is used to characterize the temporal extinction process and identify the time instant in which global flame blowout occurs. When analyzing the extinction phenomenon between the three wall configurations (diffuser, nominal, nozzle), the total duration of flame extinction was different for all cases. To account for this, a method was developed to align the time-frames between the three test conditions by developing a 
normalized time variable, $t^{*}$, which is expressed in equation 1 ; this method of temporal analysis has also been documented in previous studies of flame blowout [27].

$$
t^{*}=\frac{t-t_{s}}{t_{b}-t_{s}}
$$

Here, $t$ is the actual time, $t_{s}$ is the start of the extinction process and $t_{b}$ is the time where global blowout occurs. The time where the extinction process begins $\left(t_{s}\right)$ corresponds to the time in which the chemiluminescence intensity changes from a constant stable burning value and begins to decrease; the decreasing luminosity of the two chemiluminescence signals is indicative of the equivalence ratio decreasing through time. The chemiluminescence intensity continues to decrease until global blowout occurs. Once the flame is blown out, the chemiluminescence intensity reaches a constant, minimum value. The first time instant where the mean pixel intensity reaches this minimum value is denoted as $t_{b}$. Using this normalization method, the start of the extinction process is equivalent to $t^{*}=0$ and the time of complete blowout is equivalent to $t^{*}=1$.

\section{Results}

The following sections evaluate the effects of pressure gradient tailoring upon the mechanisms of lean flame blowout followed by a frequency and Strouhal number analysis. The three test conditions for tailoring the pressure gradient of interest are the diffuser, nominal, and nozzle configurations as described in Section 2.1. The freestream velocity for each test condition is maintained at $U_{\infty}=25 \mathrm{~m} / \mathrm{s}$, corresponding to a Reynolds number of $\operatorname{Re}=U_{\infty} H / v$ $\approx 27,000$. The blowout methodology is the same for each test configuration as described in Section 2.5.

An example of the mean $\mathrm{C}_{2}{ }^{*}$ and $\mathrm{CH}^{*}$ species intensities throughout the duration of extinction are provided within Figure 4a. The curves are
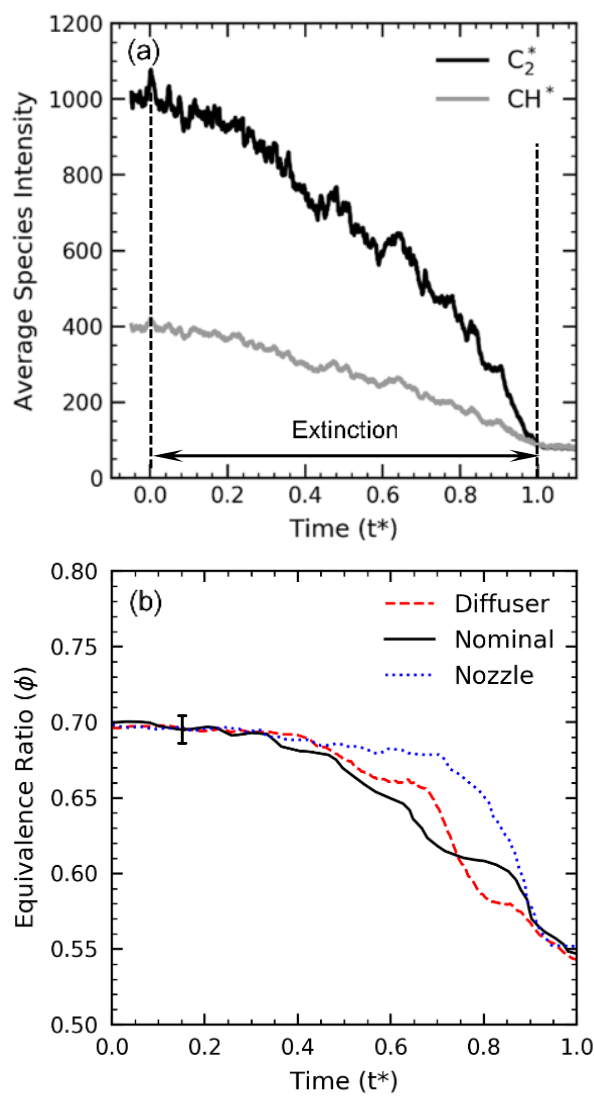

Figure 4. a) $\mathrm{C}_{2} *$ and $\mathrm{CH}^{*}$ species intensities for the nominal configuration throughout the extinction duration, and b) equivalence ratio for all test cases. All quantities are spatially averaged from $2 \leq x / H \leq 4$. 
determined by calculating the mean intensity of the $\mathrm{C}_{2}{ }^{*}$ and $\mathrm{CH}^{*}$ images between $2 \leq x / H \leq 4$. Both species intensities begin decreasing when $t^{*}=0$ and continue to drop until complete blowout occurs at $t^{*}=1$. The species intensity data is used to quantify the equivalence ratio through time, as shown in Fig. 4b. The equivalence ratio for the three extinction test cases all begin near $\Phi=0.7$ and decrease throughout the extinction event. At the time of global blowout, the equivalence ratio is near $\Phi=0.55$ for all test configurations. This temporal equivalence ratio data is used to calculate the temporal density gradient across the flame as well as the laminar flame speed; both will be discussed within the subsequent sections. The uncertainty in the equivalence ratio is determined from an error propagation which accounts for the error in the air flow rate, the error in the fuel flow rate, as well as the standard error (or standard deviation) between the calibration images of $\mathrm{C}_{2}{ }^{*}$ and $\mathrm{CH}^{*}$ for the stable lean equivalence ratios; the maximum error in the equivalence ratio is approximately $\Phi \pm 0.016$.

\subsection{Pressure Gradient Tailoring and Baroclinic Torque}

The diffuser, nominal, and nozzle configurations are implemented to obtain different axial pressure gradients within the test-section. The near-field axial pressure for the stable reacting flow is depicted in Fig. 5. The pressure curves are focused on the spatial region between $2 \leq x / H$ $\leq 4$ to isolate the critical region where flame extinction occurs [5,6,18-20]. The axial pressure curves are determined from the pressure measurements described in Section 2.1. Furthermore, the pressure presented in Fig. 5 is presented as $C_{p^{-}} C_{\mathrm{p}}(0)$, where $C_{p}=P(x) / 0.5 \rho U_{\infty}^{2}$ and $C_{\mathrm{p}}(0)$ represents the value of $C_{p}$ at the upstream measurement location nearest to the bluff-body trailing edge. This method of depicting the axial pressure within the test section is specifically chosen to align the starting location of the pressure curves for all configurations while also providing visual clarity of the varying pressure gradient magnitudes, $d P / d x$, between the three wall configurations. The nozzle

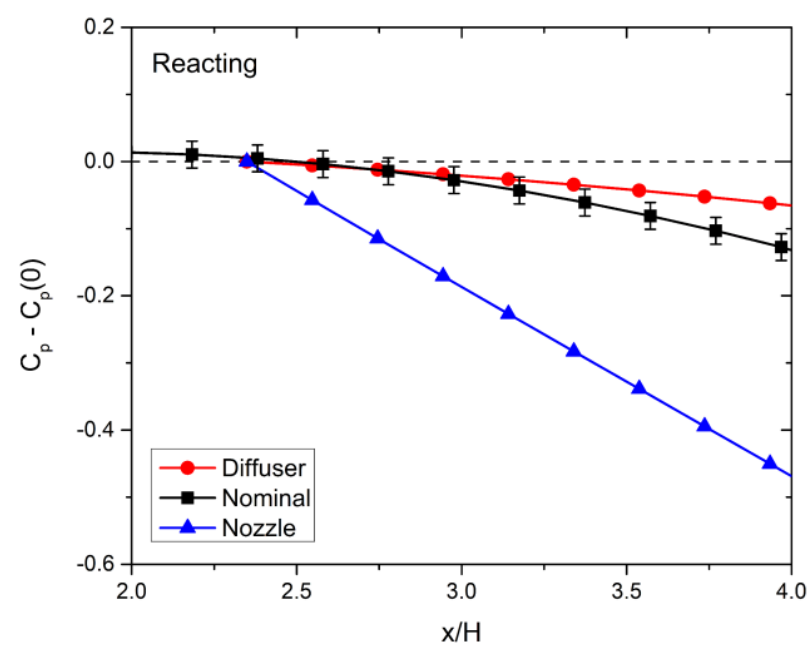

Figure 5. Pressure profiles along the axial distance of the test section. Pressure is normalized by $0.5 \rho U_{\infty}{ }^{2}$ to provide a normalized pressure coefficient. The initial pressure value $C_{p}(0)$ is subtracted from the measurements to align the pressure curves and provide visual contrast. 
configuration produces the highest-magnitude pressure gradient within the near-field domain; in contrast, the diffuser maintains the lowest magnitude pressure gradient.

The differing pressure gradients between the three wall configurations will have a direct influence on the magnitude of baroclinic torque production within the near-field domain. The baroclinic torque is expressed as $1 / \rho^{2}(\nabla \rho \times \nabla P)$, where the misalignment between density and pressure gradients imparts a rotational motion within the flow field $[4,6,22,23,25]$. The pressure gradient is determined from the static pressure measurements depicted within Fig. 5, and the density gradient is determined from:

$$
\nabla \rho=\frac{\rho_{u}-\rho_{b}}{l_{f}}\left(n_{x} \hat{i}+n_{y} \hat{j}\right)
$$

where $\rho_{u}$ and $\rho_{b}$ are the densities of the unburnt reactants and burnt products, respectively, $l_{f}$ is the laminar flame thickness, and $n_{x}$ and $n_{y}$ are the normal components of the flame. The density of the burned gas is determined from the equivalence ratio presented in Fig. 4 and the laminar flame thickness is acquired from Tang [37] and Vagelopous [38].

The baroclinic torque generated for each of the test conditions is first evaluated locally using the density and pressure gradients along the wrinkled flame structure. The local baroclinic torque is then spatially averaged over the axial distance $2 \leq x / H \leq 4$ and shown in Fig. 6. Since the baroclinic torque is calculated at the instantaneous flame location, the curves presented within Fig. 6 also account for the shift in flame position through time. Considering that the nozzle case has the highest pressure gradient, the baroclinic torque is the highest for the nozzle and the lowest for the diffuser, Fig. 6. The baroclinic torque is relatively constant with time for both the nozzle and nominal cases, but begin to experience a significant drop in magnitude around $t^{*}=0.75$. For the diffuser configuration, the baroclinic torque remains at a nominally constant small value for the

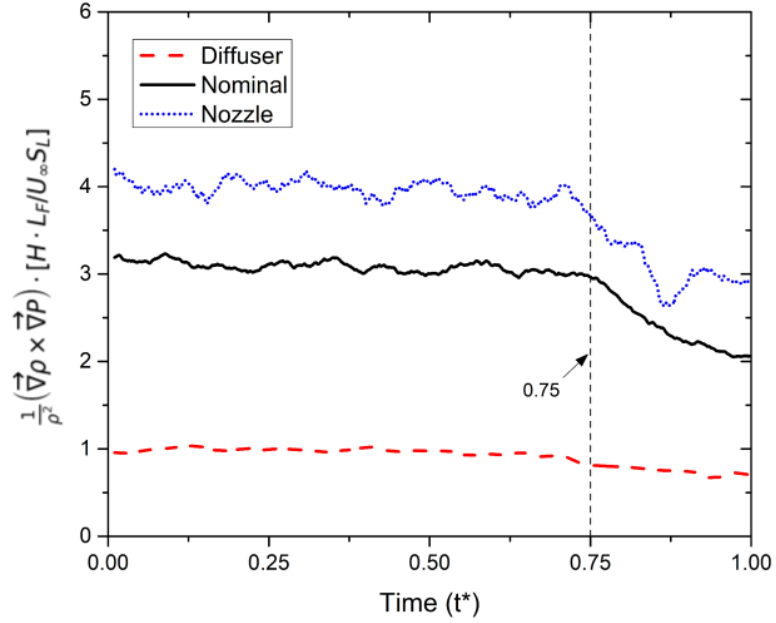

Figure 6. Temporal evolution of baroclinic torque throughout the duration of extinction for the three different wall configurations. The baroclinic torque is normalized by $\left(H \cdot L_{F}\right) /\left(U_{\infty} \cdot S_{L}\right)$ and spatially averaged between $2 \leq x / H \leq 4$. 
majority of the extinction duration, but a slight decrease is also noticed near $t^{*}=0.75$.

\subsection{Flame and Flow Field Visualizations}

Instantaneous vorticity fields with the overlapping flame structures for the diffuser, nominal, and nozzle configurations are presented within Fig. 7 to visualize the influence of baroclinic torque on the flame-flow field. The contours are focused on the critical region for flame blowout, $2 \leq x / H \leq 4$. Additionally, the local flame strain rate $(\kappa)$ is superimposed on the flame boundaries shown within Fig. 7. The local strain rate is calculated using equation 3, where $u$ and $v$ are the stream-wise and cross-stream velocity components, respectively.

$$
\kappa=-n_{x} n_{y}\left(\frac{\partial u}{\partial y}+\frac{\partial v}{\partial x}\right)+\left(1-n_{x}^{2}\right) \frac{\partial u}{\partial x}+\left(1-n_{y}^{2}\right) \frac{\partial v}{\partial y}
$$

Four various time instances are provided to highlight the temporal evolution of the flame-
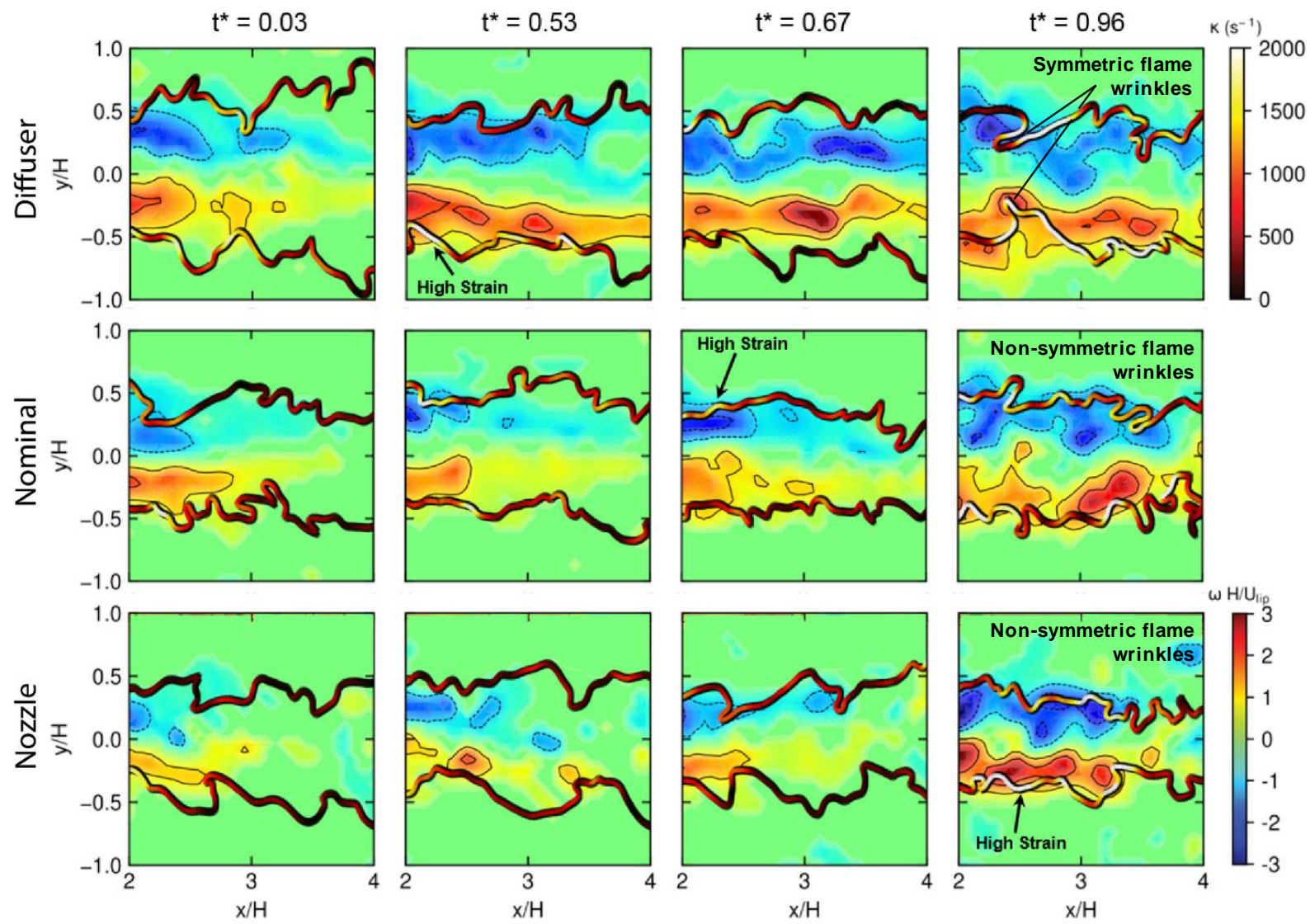

Figure 7. Instantaneous vorticity contours along with the flame structure and the local flame strain rate. Time evolution is from left to right with $t^{*}=0$ representing the beginning of the extinction process and $t^{*}=1$ representing the time of complete blowout.

flow field. The first time instant is indicative of a stable burning flame and is selected at $t^{*}=$ 
0.03 just after the flame extinction process is initiated. The second and third frames show the phenomena during the extinction process, and the last frame depicts the flame just prior to complete blow-out at $t^{*}=0.96$.

During stable combustion, the flame structure is wrinkled and is generally symmetrical across the bluff-body centerline $(y / H=0)$. The flame edge extends beyond the regions of highmagnitude shear layer vorticity near the beginning of the extinction process, however, the vorticity field for low baroclinic torque (diffuser) occupies the largest area within the domain compared to the other configurations. The expansive appearance of the low baroclinic torque case is due to the larger volume afforded by the diffuser within the downstream locations. As the flame proceeds towards complete blowout, the flame structure begins to overlap with regions of high-magnitude shear layer vorticity for all configurations. Near blowout, it is noted that the flame within the diffuser configuration maintains symmetrical wrinkling structures across the bluff-body centerline (Fig. 7), opposed to the nominal and nozzle configurations where the overall symmetry of the flame is completely lost.

In addition, the magnitude of vorticity increases and occupies a greater area within the domain as the flame approaches global blowout. To evaluate the temporal vorticity dynamics, the vorticity characteristic length scales are defined in Fig. 8. Note that it is challenging to determine the recirculation zone length from the instantaneous reacting flow field data, therefore, the shear layer length is used. The shear layer dimensions are dependent on the recirculation zone dynamics and the density ratio across the flame [23]. The shear layer length is determined as the axial distance from the trailing edge of the bluff-body to the point where the shear generated vorticity reaches a minimum; likewise, the shear layer width is calculated as the vertical length from the bluff-body centerline to the height in which the vorticity reaches zero. In all of the cases, there is a subtle increase in the shear layer length with time, however, the shear length for the nozzle condition is significantly shorter than the diffuser and nominal cases, as presented within Fig. 8a. Likewise, the shear layer widths for the diffuser and nominal cases are similar, however, past the time $t^{*}=0.45$, the width of the diffuser case begins to deviate and increases over the nominal case, Fig. 8b. For the nozzle case, the shear width is relatively constant until approximately $t^{*}=0.75$, where a sudden increase is noticed. The shear layer dimensions are smallest for the nozzle and largest for the diffuser, as reflected by the expansive vorticity field captured within Fig. 7. When comparing the three test conditions, the shear 
dimensions are inversely proportional to the magnitude of baroclinic torque production; in other words, the baroclinic torque production attenuates the shear layer vorticity due to its opposing orientation [26,27].

The mean transverse flame position from the bluff-body centerline, spatially averaged over the axial distance of $2 \leq x / H \leq 4$, is presented within Fig. 8b. It is noticed that the diffuser maintains the widest flame brush, while the nominal and nozzle configurations have similar widths until about $t^{*}=0.75$, at which point the flame brush begins to narrow. It is also noteworthy that the mean flame position is representative of the spatial location where baroclinic torque is generated through time. During the first half of the blowout process, the mean transverse flame position is greater than the width of the shear layer, ensuring that the flame is maintained away from the high-magnitude shear layer vorticity; this reflects the observations of the flame edge bounding the shear layers in Fig. 7. During the late stages of the extinction process, past the time $t^{*}=0.75$, it becomes evident that the flame is inducted toward the bluffbody centerline, meaning that the spatial location where baroclinic torque is generated also approaches the shear layer region. Despite the spatial convergence of the two vorticity mechanisms, the shear layer width broadens through time as seen within Fig. 8b. The temporal increase of the shear layer width suggests that the magnitude of baroclinic torque, rather than spatial location where it is produced, has the strongest influence on the shear layer vorticity dynamics.

Near blowout, flame position and the shear layer widths are seen to overlap with Fig $8 \mathrm{~b}$, indicating that there are strong shear layer and flame interactions near blowout. This is also consistent with the overlapping flame and vorticity fields presented in the late stages of the extinction process in Fig. 7. The flame and shear layer interaction dynamics have been
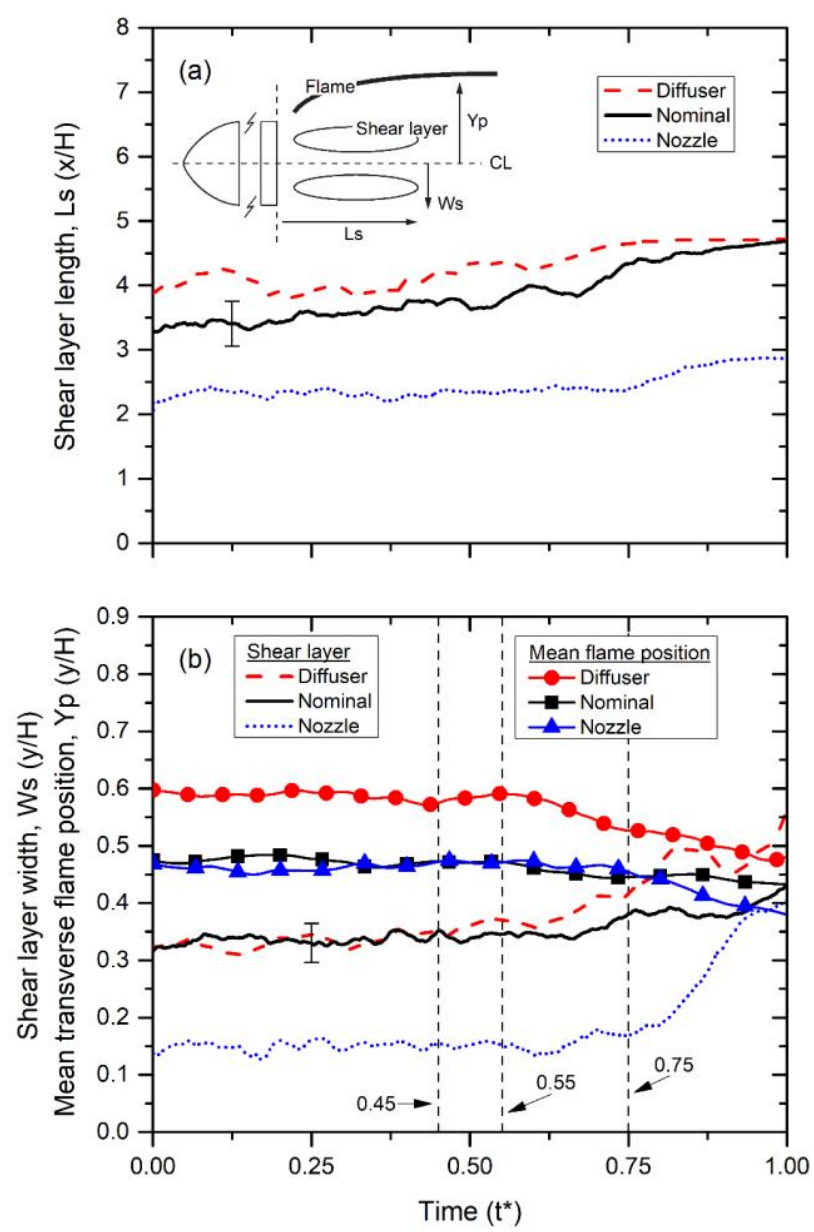

Figure 8. a) Shear layer length and b) shear layer width and mean transverse flame position through time. 
previously described as the source of increased hydrodynamic strain along the flame and is, therefore, a key contributor to the mechanisms of flame blowout [5,6,19,20,27]. When comparing the severity of the shear layer and flame interaction between the three configurations, the flame position for the diffuser configuration decreases more rapidly than the other two configurations while the shear layer width increases at the highest rate compared to the other configurations. This indicates that the lower magnitudes of baroclinic torque produced from the diffuser enhance the temporal flame-shear layer vorticity interaction and consequently accelerates flame blowout. In contrast, the nozzle configuration delays the flame and shear layer interaction and is therefore expected to attenuate the overall mechanisms of flame blowout.

\subsection{Flame-Vorticity Interaction Dynamics}

The shear layer vorticity and the baroclinic torque are the two main sources of vorticity that play a vital role in the flame stabilization and extinction processes. The shear layer vorticity is generated from the no-slip condition at the bluff-body while the baroclinic torque is produced by the presence of the flame within the flow domain. These two sources of vorticity act in opposing directions and remain separated as long as there is sufficient heat release to maintain the baroclinic torque production along the flame boundary. The nozzle configuration has been shown to produce the largest magnitudes of baroclinic torque, however, in the presence of the shear layer vorticity, the two opposing vorticity mechanisms are expected to attenuate each other, and thus the overall magnitudes of vorticity are decreased [26]. Therefore, the delayed flame-shear layer interaction observed from the nozzle configuration is due to the decreased magnitudes of shear layer vorticity due to the attenuation effect of the higher baroclinic torque production. The vorticity magnitudes within the shear layer are compared to the vorticity magnitudes experienced at the location of the flame boundary (denoted as flame vorticity) within Fig. 9. The shear layer vorticity is extracted from the vorticity field along the line of maximum vorticity within the recirculation zone and reattachment regions. The vorticity experienced by the flame is extracted along the flame boundary. As observed in Fig. 7, it is expected that the vorticity experienced at the location of the flame boundary is less than the vorticity within the shear layer; this is now quantified in Fig. 9. During the first half of the extinction process, the nozzle configuration experiences the lowest magnitudes of shear layer and flame vorticity. This is due to the increased baroclinic torque magnitudes and the corresponding attenuation effect it has on the shear layer vorticity. 

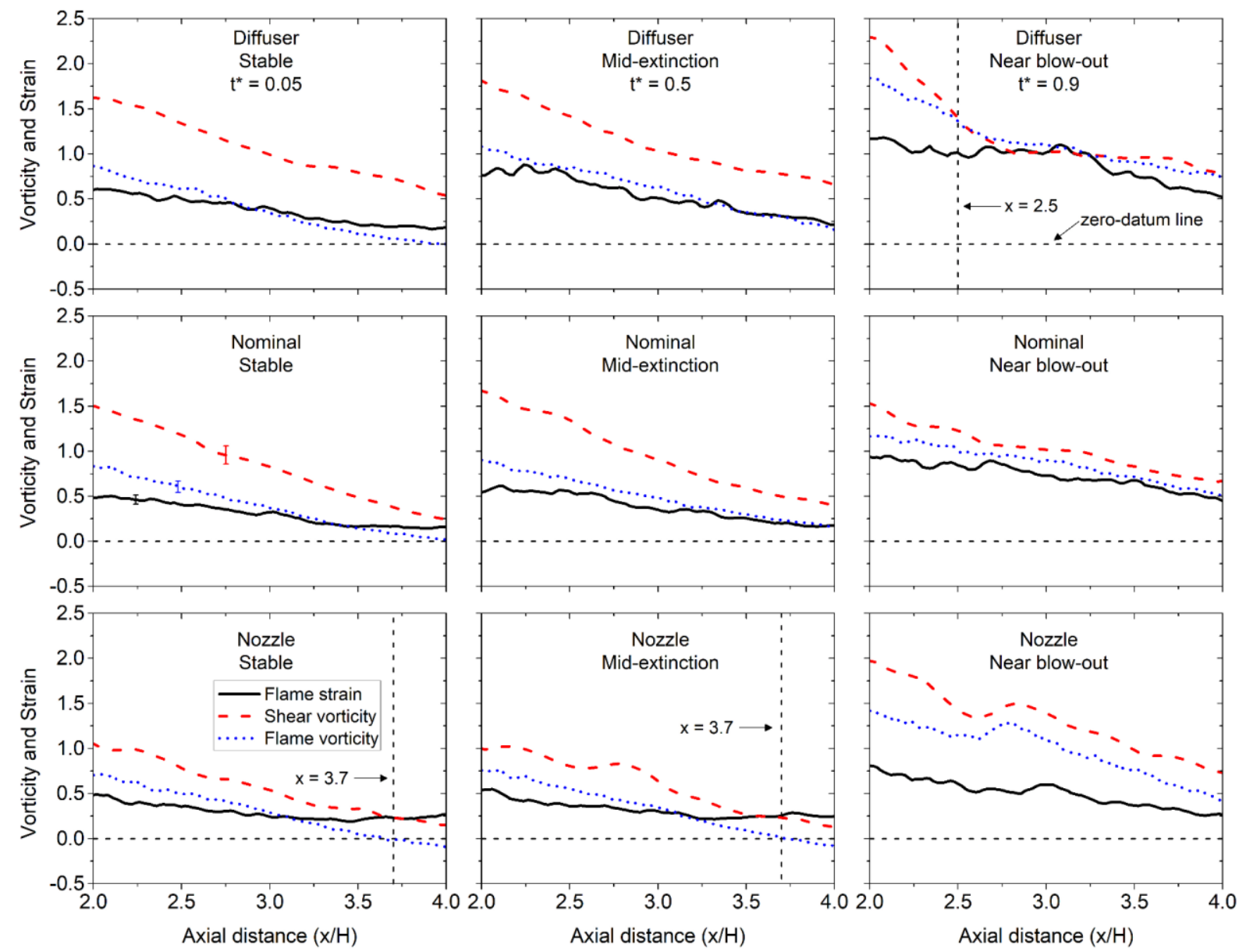

Figure 9. Axial profiles of the shear layer vorticity, vorticity along the flame, and flame strain rate. All quantities are normalized by $H / U_{\infty}$.

Moreover, the shear layer and flame vorticity both decrease along the axial distance for all test section configurations. The decreasing vorticity magnitudes are a result of the dampening effects from baroclinic torque, however, dilatation and viscous dissipation vortex mechanisms also contribute to the diminishing vorticity magnitudes within the near-field domain [4,6,24]. For all test cases, the decreasing vorticity magnitudes approach the zero-datum line; however, the vorticity experienced at the location of the flame boundary switches sign at $x / H=3.7$ for the nozzle configuration and sustains a negative value for the first half of the extinction duration. The transition from positive to negative vorticity magnitudes is indicative of the baroclinic torque becoming the dominant vorticity mechanism within the flow field and eliminating the shear layer vorticity downstream of the bluff-body. This phenomenon is consistent with previous studies of reacting bluff-body flow fields that have highlighted the increasing dominance of 
baroclinic torque as the axial distance from the bluff-body is increased [6,22,23,27]. For the diffuser and the nominal cases, it is expected for the flame-generated vorticity to switch sign further downstream of the bluff-body at distances $x / H>4$ as the influence of baroclinic torque is expected to dominate.

Furthermore, as the flame approaches blowout, the difference between shear layer and the flame-generated vorticity decreases indicating a prominent flame-shear layer interaction near blowout. The difference in

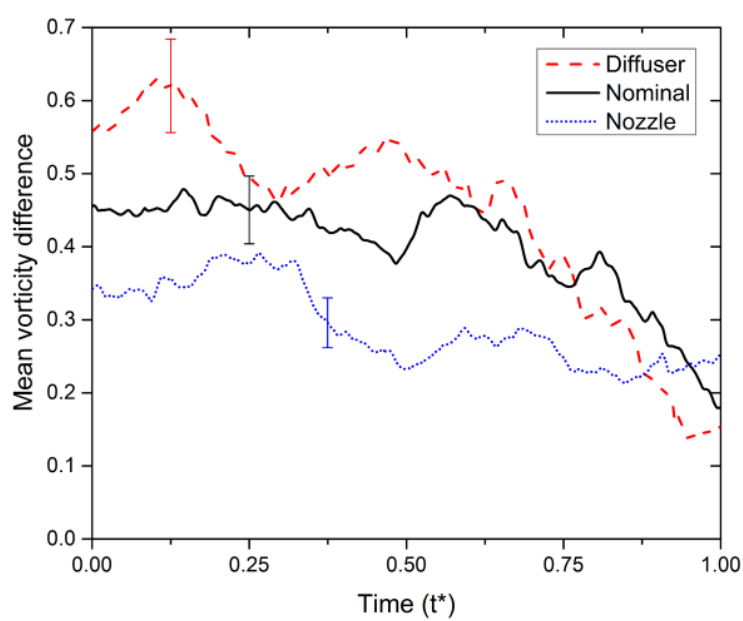

Figure 10. Mean difference between the shear layer vorticity and freestream vorticity at the location of the flame boundary. Values are normalized by $H / U_{\infty}$. magnitudes between the flame and shear layer vorticity are quantified within Fig. 10. The vorticity difference is determined by taking the difference between the instantaneous mean vorticity within the shear layer and along the flame within the spatial domain between $2 \leq x / H \leq$ 4 for every instant in time and portrayed in Fig. 10 as a non-dimensional quantity $\Delta \omega H / U_{\infty}$. Throughout most of the extinction duration, the mean difference between the shear layer and flame vorticity magnitudes is smallest for the nozzle configuration and largest for the diffuser. Considering that the baroclinic torque and shear layer vorticity have opposing signs, the low baroclinic torque production for the diffuser has the weakest attenuation of the shear layer vorticity. The lack of vorticity attenuation combined with the large flame span experienced by the diffuser results in the largest difference between vorticity magnitudes, as shown within Fig. 10. Conversely, the nozzle has the strongest dampening effect upon the shear layer, resulting in a subtle difference in vorticity magnitudes. Throughout the duration of the extinction process, the mean vorticity difference between the flame and shear layer decreases for all test cases which reflects that at blow-out, the shear layer and flame-generated vorticity magnitudes are similar and thus provides additional support to the flame-shear layer interaction as a precursor to blowout.

During the lean blowout process, the equivalence ratio decreases monotonically and the flame temperature decreases. The temperature decrease results in decreased density ratio across the flame between the burned and unburned gases. Since the vorticity dilatation and flamegenerated vorticity scale with the density ratio, the decrease in the density ratio will weaken the 
effect of attenuating the shear layer vorticity by the action of vorticity dilatation and flamegenerated vorticity, i.e., the shear layer vorticity is unabated and dominates the whole flow field. The magnitude of the shear layer vorticity increases near the bluff-body and broadens into the wake along the axial distance [5]. Furthermore, as the equivalence ratio is reduced, the diminishing magnitudes of baroclinic torque are coupled with decreased flame speeds, allowing the flame to interact with the high-magnitude shear layer vorticity. Within this study, the baroclinic torque produced by the nozzle configuration is the largest in view of mutual vorticity attenuation and hence, it is expected to attenuate the flame-vortex interaction mechanism driving a delayed blowout. Conversely, the diffuser configuration experiences the largest decrease in the vertical flame position through time coupled with the largest shear layer vorticity magnitudes. Therefore, the interaction between the shear layer and the flame occurs earliest for the diffuser and will lead to blowout quicker than the other configurations. In addition, near the end of the extinction process, the strain rate along the flame is largest for the diffuser (see diffuser in Fig. 9), suggesting that the strain rate along the flame is a critical parameter indicative for flame blowout.

\subsection{Strain Rate Evolution and Karlovitz}

\section{Number Analysis}

The hydrodynamic strain rate along the flame is an important parameter for characterizing localized extinctions along the flame $[18,19,27,39]$. The strain rate
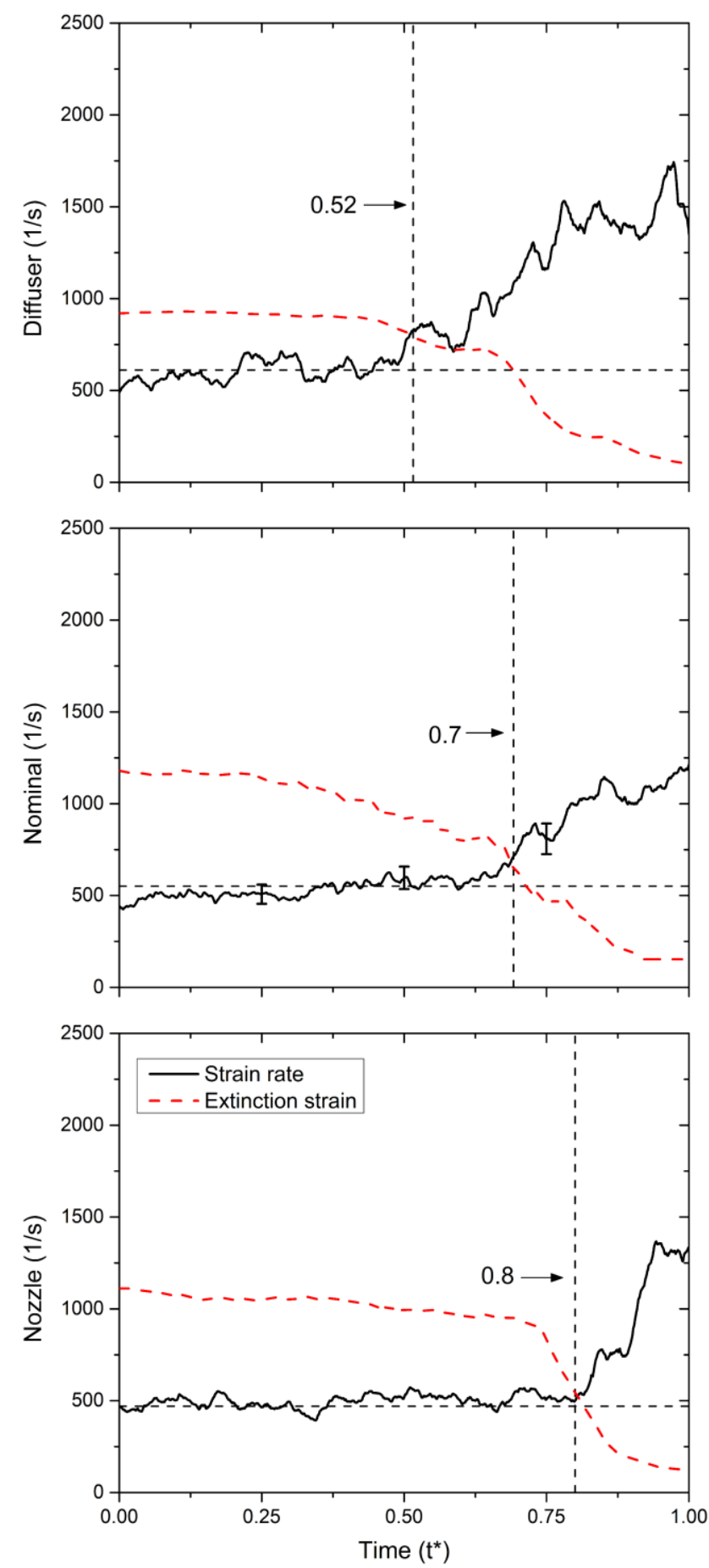

Figure 11. Temporal strain rate along the flame and extinction strain rate limit. 
along the flame is calculated using equation 3 for each of the wall configurations and presented within Fig. 11. Similar to the analysis within the previous sections, the strain rate is spatially averaged between $2 \leq x / H \leq 4$ to provide a global perspective of the temporal strain rate evolution within the critical domain for lean blowout. For all configurations, the strain rate increases through time, however, there is a time period during the beginning of the extinction process where the strain rate is relatively constant; this is represented by the horizontal line within Fig. 11. After maintaining relative constancy, the strain rate undergoes rapid growth for all configurations. It is also noted that the time duration to maintain a nearly constant strain rate along the flame varies for the three test conditions; this time duration is also depicted within Fig. 11. The high-magnitude baroclinic torque production for the nozzle allows the strain rate along the flame to maintain a nominally constant value until $t^{*} \approx 0.8$. Beyond this time instant, the strain rate undergoes rapid temporal growth and global blowout ensues. For the diffuser and nominal configuration, the significant increase in the strain rate appears earlier in the extinction process, at $t^{*} \approx 0.52$ and $t^{*} \approx 0.7$, respectively. This indicates that higher magnitudes of baroclinic torque are able to hinder the shear-induced mechanisms of extinction. Furthermore, it is important to note that the point where the strain rate experiences a rapid increase closely corresponds to the time instant where the flame strain rate is equivalent to the extinction strain rate limit. The extinction strain rate within Fig. 11 is obtained using CHEMKIN [40] for premixed opposed-jet flames, and is evaluated as the maximum feasible velocity gradient in which a flame can be sustained for a given equivalence ratio and flame thickness; this approach has also been implemented in previous work to analyze the dynamics of lean flame blowout $[27,41]$.

In view that excessive strain rates lead to localized flame extinctions, early onset of the strain rate experienced within the diffuser case indicates an expedited flame blowout. For example, considering that there is a general agreement that the competition between the fluid mechanical and chemical kinetic processes has a direct influence on the blowout process, the competing time

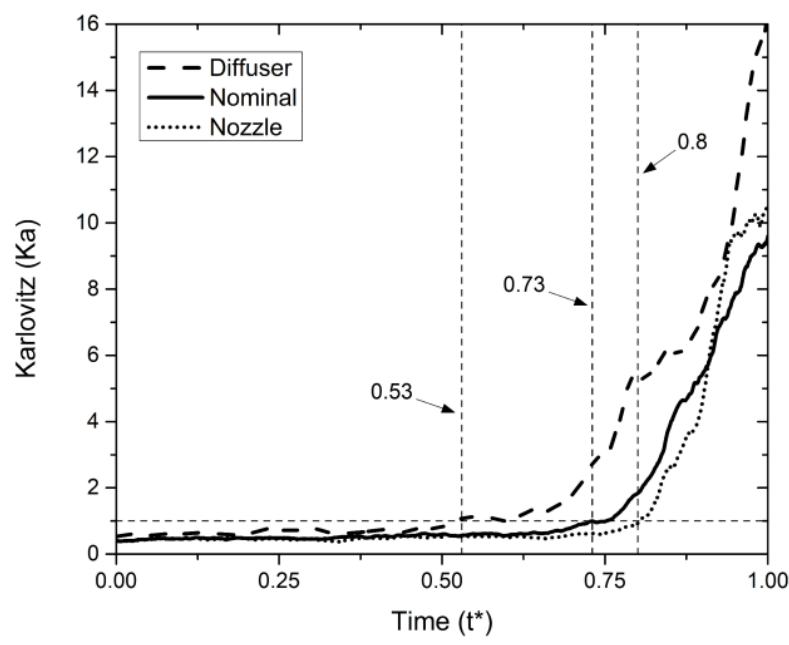

Figure 12. Karlovitz number curve for the three test section configurations. $K a=1$ is used as a reference to indicate the initiation of local extinctions along the flame. 
scales play a key role [5]. To quantify this, the Karlovitz number, $\mathrm{Ka}$, which is calculated as the ratio of the flame strain rate to the extinction strain rate tolerance is presented in Fig. 12. From a heat and mass transfer perspective, the Karlovitz number represents the ratio of chemical timescales to the hydrodynamic timescales. Thus, if $K a<1$ a stable reaction can be sustained, and if $K a>1$ indicates the flame is extinguishing. However, $K a=1$ is used as a reference marker and does not suggest immediate blowout. A Karlovitz number of unity is associated with localized extinctions where an unsteady flame can persist through self-healing and re-ignition of flame holes [6]. Regardless of the magnitude of baroclinic torque, the Karlovitz number is less than unity through the first half of the extinction process, Fig. 12. At the time instant of $t * \approx 0.53$, the Karlovitz number for the diffuser case increases rapidly, followed by the nominal and nozzle configurations at $t^{*} \approx 0.73$ and $t^{*} \approx 0.8$, respectively. This indicates that the increased production of baroclinic torque from the nozzle configuration elongates the time required for the Karlovitz number to reach unity. In this manner, the nozzle delays the initiation of localized extinctions along the flame. For all three test configurations, it is also noticed that the sharp rise in the flame strain rate occurs near the same time as when the shear layer width and mean transverse flame position begin to converge toward one another, as previously shown within Fig. 8. This behavior implies that the sudden increase in the Karlovitz number can be used as a marker for the flameshear layer interaction mechanism that drives global flame extinction.

\subsection{Baroclinic Influence on Hydrodynamic Instabilities}

Throughout the extinction process, the flame structure oscillates with a distinct frequency. Previous studies have demonstrated that flame structures and wrinkling are heavily driven by local vorticity dynamics $[27,34,42,43]$. For this reason, it is anticipated that the flow field undergoes similar oscillations as the flame. To quantify the flame and flow instabilities, the power spectral density (PSD) of the flow field is provided within Fig. 13. The PSD is evaluated using the transverse velocity within the shear layer to capture the flow oscillations with minimal contributions from the stream-wise acceleration of the combustion products. The transverse velocity signal is probed at the spatial location where it is maximum; the frequency analysis is then performed using Welch's overlapping segment averaging estimator [44].

During stable combustion, the BVK frequency for the nominal configuration is approximately $450 \mathrm{~Hz}$. As the flame progresses towards blowout, there is a clear shift in the 
frequency to approximately $340 \mathrm{~Hz}$, which is the expected BVK frequency for a non-reacting bluff-body flow [6]. As the flame blows-out, the general trend of the BVK frequency shift is similar for the diffuser and nozzle configurations. However, the BVK frequency and its harmonics are not identical between the three test-section configurations.

To further supplement the analysis of hydrodynamic instabilities throughout the extinction process, a Strouhal number analysis is conducted. The Strouhal number, $S_{t}$, is a dimensionless number that is used to provide a characteristic description of flow oscillations. For bluff-body flows, the Strouhal number is defined as:

$$
S_{t}=\frac{f L}{U_{\infty}},
$$


where $f$ is the oscillatory frequency, $L$ is a characteristic length, and $U_{\infty}$ is the freestream velocity. For non-reacting bluff-body flows, the characteristic length is commonly selected as the height of the bluff-body $(H)$ to provide a unified scaling criterion to describe the anticipated BVK vortex shedding frequency [4]. However, for extinguishing flames, changes in the density ratio and flame position cause the BVK frequency and its associated harmonics to decrease, as demonstrated in Fig. 13. For this reason, a dynamic characteristic length scale should be chosen to accommodate for the frequency shift and allow for a singular Strouhal number to be obtained. Since the frequency analysis within Fig. 13 depicts the velocity fluctuations within the shear layer, the length scale selected should also come from this region. When comparing the shear layer dimensions within Fig. 8, the shear layer length appears to capture the effects of the pressure gradient tailoring better than the shear layer width; there is a clear seperation in shear layer lengths between the three test cases. For this reason, the shear layer length is chosen as an appropriate characteristic length for the
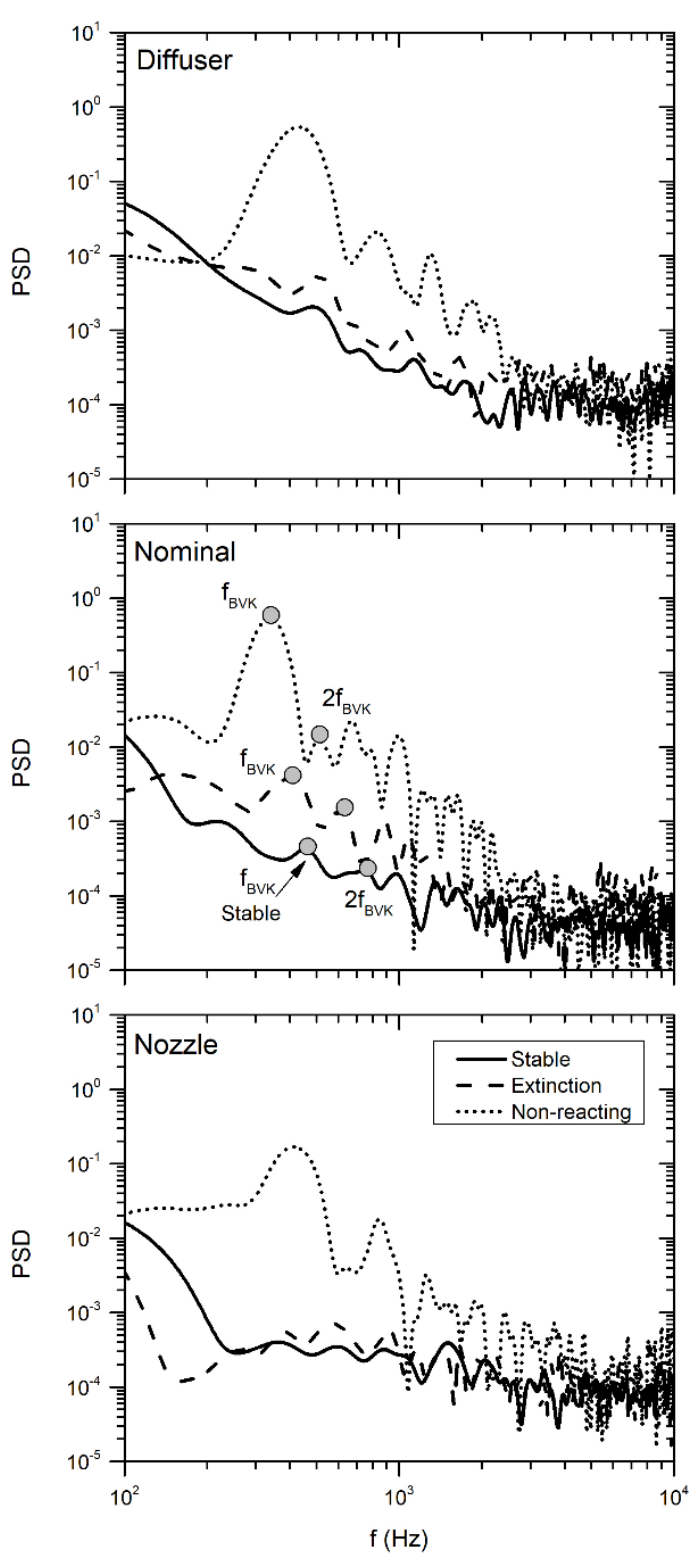

Figure 13. Frequency spectra of the transverse (span-wise) velocity showing the BVK and harmonic frequency shift throughout extinction. 
Strouhal number analysis [45]. It is important to note that the shear layer length is proportionally related to the length of the recirculation zone. The time-averaged recirculation zone and shear layer lengths for a stable lean flame at $\Phi=0.7$ and a nonreacting condition of $\Phi=0$ shows that the shear layer length is nominally $48 \%$ longer than the length of the recirculation zone. This indicates that the shear layer length captures the effects of the changing density ratio in the same manner as the recirculation zone discussed in literature $[23,46]$. The shear layer length is also beneficial for capturing turbulence and viscous mixing effects which will influence the stability of the flame. It has also been shown to provide adequate scaling results within previous studies of blowout [27].

The Strouhal number is evaluated throughout the duration of extinction for the three testconditions and presented within Fig. 14. The Strouhal number is evaluated two separate ways: the first is a traditional method utilizing the bluff-body height as the characteristic length and the BVK frequency [6,23], as shown in Fig. 14a. The second method employs the shear layer length $(L)$ as the characteristic length and is coupled with the first harmonic of BVK $\left(2 f_{B V K}\right)$, as shown in Fig. 14b. To be more specific, the first BVK harmonic is used as it closely matches the oscillatory frequency of the flame throughout the extinction duration $(\sim 600-800 \mathrm{~Hz})$, and thus, best represents the flame-flow field instabilities [27]. Figure $14 \mathrm{a}$ demonstrates that the traditional Strouhal number decreases as the equivalence ratio decreases. This is a result of the BVK frequencies decreasing through time as presented within Fig. 13. Once extinguished, the nonreacting Strouhal number for the nominal configuration is approximately 0.22 , which is the expected number for nonreacting bluff-body flows [6,23]. It is also noteworthy that the nonreacting Strouhal number for the nozzle and diffuser configurations are slightly
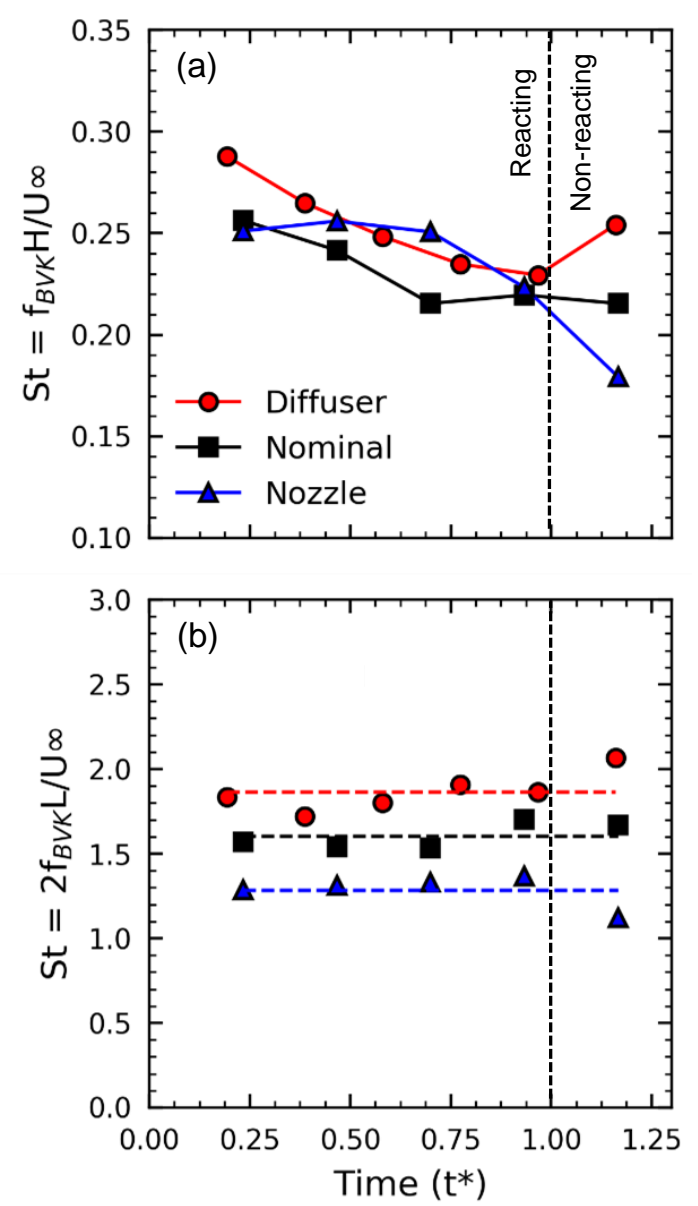

Figure 14. Strouhal number for various test section configurations. (a) Strouhal number calculated using BVK frequency and bluffbody height. (b) Scaled Strouhal number using the first BVK harmonic frequency $\left(2 f_{B V K}\right)$ and the shear layer length $(L)$. 
offset from the nominal configuration due to variations in flow confinement [6].

In contrast, Figure 14b shows the Strouhal number remains nominally constant for all three test configuration; this is even true for the non-reacting flow field which is also included in Fig. 14 as the data point residing beyond $t^{*}=1$. The unified Strouhal number indicates that the oscillating frequencies within the flow field are coupled with the extinguishing flame-flow dynamics. Although a unified Strouhal number scaling achieved for each configuration, there is a slight difference between the three test configurations (1.9 to 1.3). The diffuser configuration exhibits the highest Strouhal number of $S_{t} \approx 1.9$ while the nozzle case exhibits the smallest value of $S_{t} \approx 1.3$. This implies that the Strouhal number is more sensitive to the shear layer length than it is to the oscillatory frequency. With respect to the combustion-induced vorticity mechanisms, an increase in baroclinic torque production leads to a decreased Strouhal number due to the effect of shear layer vorticity attenuation.

\section{Conclusion}

The influence of pressure gradient tailoring on the temporal mechanisms of bluff-body flame extinction was investigated in a high-speed combustion facility. The axial pressure gradient was varied by manipulating the test-section wall geometry between three configurations: a diffuser, nominal, and nozzle section. For all configurations, the results confirm that flame-vortex interactions are the primary mechanism of extinction for bluff-body flames. As the equivalence ratio decreased through time, the flame speed reduced and the flame boundary interacts with the shear layer regions. This interaction was shown to increase the strain rate along the flame and ultimately lead to global blowout.

The validity of the extinction mechanism was also augmented by analyzing variations in flame-vortex interactions across the three wall configurations. The differing geometry between the three test cases were shown to influence the magnitude of the axial pressure gradient, which ultimately impacted the baroclinic torque production along the flame. The increased baroclinic torque magnutudes for the nozzle configuration resulted in the lowest magnitude shear layer vorticity due to a mutual vorticity attenuation. The decrease in shear layer vorticity mitigated the interaction between the flame and the shear layer, and resulted in lower strain rates along the flame throughout the duration of extinction. The opposite was true for the diffuser configuration, where the lowest magnitudes of baroclinic torque resulted in the largest shear layer vorticity 
magnitudes. The increased shear layer magnitudes accentuated the flame-vorticity interactions, resulting in the largest strain rate magnitudes along the flame throughout the duration of extinction.

To further discern the temporal variations of flame-vorticity interactions, Karlovitz numbers were analyzed to predict the time duration required for localized extinctions to initiate along the flame boundary. Similar to the strain rate analysis, the time required for the Karlovitz number to reach a critical value of unity was delayed for the nozzle configuration and shortened for the diffuser. Additionally, the time instant in which the Karlovitz number surpasses a value of unity was observed to coincide with the time where the mean transverse flame position and shear layer length began to converge. It is shown that this time instant is when hydrodynamic strain rate exceeds the flame extinction strain rate limit. This indicates that the sudden rise of the Karlovitz number can be used as an indicator of the flame and shear layer interaction dynamics.

Finally, the magnitude of baroclinic torque was found to have minimal effects upon the BVK and harmonic frequencies. For all test conditions, the BVK frequency decreases through time and converges toward the expected frequency for non-reacting bluff-body flows. To further investigate the hydrodynamic instabilities throughout extinction, a Strouhal number was evaluated temporally for all test configurations. Using the frequency of the first BVK harmonic $\left(2 f_{B V K}\right)$ and the shear layer length as the characteristic length, a constant Strouhal number was obtained for each independent test case. However, a variation in the Strouhal number value was found between the three cases and was attributed to the attenuation effect of the baroclinic torque influence on the shear layer length and vorticity magnitudes. In this manner, increased baroclinic torque production led to lower Strouhal numbers due to reduced shear layer length scales. 


\section{Acknowledgments}

The work is sponsored by the Air Force Office of Scientific Research (FA9550-16-1-0403 and FA9550-16-1-0044, Program Manager: Dr. Chiping Li). This material is based upon work supported by the Air Force Office of Scientific Research under award numbers FA9550-16-10403 and FA9550-16-1-0044. The authors would like to acknowledge Dr. Campbell Carter at the AFRL for providing equipment support for performing these experiments.

\section{References}

[1] H. Ebrahimi, Overview of Gas Turbine Augmentor Design, Operation, and Combustion Oscillation, 42nd AIAA/ASME/SAE/ASEE Joint Propulsion Conference \& Exhibit (2006). paper 2006-4916.

[2] J. Lovett, T. Brogan, D. Philippona, B. Keil, T. Thompson, Development Needs for Advanced Afterburner Design, 40th AIAA/ASME/SAE/ASEE Joint Propulsion Conference \& Exhibit (2004). paper 2006-4916.

[3] D. Dunn-Rankin, Lean Combustion: Technology and Control, Academic Press, Burlington, MA, 2008.

[4] T. Lieuwen, S.J. Shanbhogue, S. Khosla, C. Smith, Dynamics of Bluff Body Flames Near Blowoff, 45th AIAA Aerospace Scienes Meeting \& Exhibit (2007). paper 2007-169.

[5] S. Nair, T. Lieuwin, Near-Blowoff Dynamics of a Bluff-Body Stabilized Flame, J. Propuls. Power 23 (2007) 421-427.

[6] S.J. Shanbhogue, S. Husain, T. Lieuwen, Lean Blowoff of Bluff Body Stabilized Flames: Scaling and Dynamics, Prog. Energy Combust. Sci. 35 (2009) 98-120.

[7] E.E. Zukoski, F.E. Marble, Experiments concerning the mechanism of flame blowoff from bluff bodies, Gas Dynamic Symposium on Aerothermochemistry (1956), p. 205-210.

[8] J.P. Longwell, E.E. Frost, M.A. Weiss, Flame Stability in Bluff Body Recirculation Zones, Ind. Eng. Chem. 45 (953) 1629-1633.

[9] J.P. Longwell, J.E. Chenevey, W.W. Clark, E. Frost, Flame stabilization by baffles in a high velocity gas stream, Proc. Combust. Inst. 3 (1948) 40-44.

[10] A. Roshko, On the Wake and Drag of Bluff Bodies, J. Aeronaut. Sci. (1955) 124-132.

[11] R.I. Ozawa, Survey of basic data on flame stabilization and propagation for high speed combustion systems, OH, 1971. Technical Report TR-70-81.

[12] M.V. Herbert, Aerodynamic influences on flame stability, Prog. Combust. Sci. Technol. 
(1960) 61-109.

[13] A.H. Lefebvre, D.R. Ballal, Gas Turbine Combustion, CRC Press, Boca Raton, FL, 1998.

[14] N. Peters, Turbulent Combustion, Cambridge University Press, Cambridge, U.K., 2000.

[15] H.M. Nicholson, J.P. Field, Some experimental techniques for the investigation of the mechanism of flame stabilization in the wakes of bluff bodies, Symp. Combust. Flame Explos. Phenom. 3 (1948) 44-68.

[16] S.S. Penner, F. Williams, Recent studies on flame stabilization of premixed turbulent gasses, Appl. Mech. Rev. 10 (1957) 229-237.

[17] G. Winterfield, On processes of turbulent exchange behind flame holders, Symp. (Int.) Combust. 10 (1965) 1265-1275.

[18] B.R. Chowdhury, B.M. Cetegen, Effects of free stream flow turbulence on blowoff characteristics of bluff-body stabilized premixed flames, Combust. Flame 190 (2018) 302-316.

[19] S.G. Tuttle, S. Chaudhuri, K.M. Kopp-Vaughan, T.R. Jensen, Lean blowoff dynamics of assymetrically-fueled bluff body-stabilized flames, Combust. Flame 160 (2013) 16771692.

[20] S. Chaudhuri, S. Kostka, M.W. Renfro, B.M. Cetegen, Blowoff dynamics of bluff body stabilized turbulent premixed flames, Combust. Flame 157 (2010) 790-802.

[21] P.E. Hamlington, A.Y. Poludnenko, E.S. Oran, Interactions between turbulence and flames in premixed reacting flows, Phys. Fluids 23 (2011) 1-20.

[22] P. Mehta, M. Soteriou, Wake dynamics of bluffbody stabilized premixed combustion effects of exothermicity and forcing, 41st AIAA Aerospace Sciences Meeting \& Exhibit (2003), paper 2003-835.

[23] B. Emerson, J. O'connor, M. Juniper, T. Lieuwen, Density ratio effects on reacting bluffbody flow field characteristics, J. Fluid Mech. 706 (2012) 219-250.

[24] M.K. Geikie, Z.R. Carr, K.A. Ahmed, D.J. Forliti, On the flame-generated vorticity dynamics of bluff-body-stabilized premixed flames, Flow Turbul. Combust. 99 (2017) 487-509.

[25] M.K. Geikie, K.A. Ahmed, Pressure-gradient tailoring effects on the turbulent flamevortex dynamics of bluff body premixed flames, Combust. Flame 197 (2018) 227-242.

[26] B. Morton, The generation and decay of vorticity, Geophys. Astrophys. Fluid Dyn. 28 
(1984) 277-308.

[27] A.J. Morales, I.M. Lasky, M.K. Geikie, C.A. Engelmann, K.A. Ahmed, Mechanisms of Flame Extinction and Lean Blowout of Bluff Body Stabilized Flames, Combust. Flame 203 (2019) 31-45.

[28] J. Reyes, R.K.A. Kailasanathan, Relationship between the chemiluminescence intensity rato of $\mathrm{C}^{*}$ and $\mathrm{CH}^{*}$, charge pressure, and gasoline, Energy and Fuels 32 (2018) 1093310940.

[29] W. Thielicke, E.J. Stamhuis, PIVlab - Towards user-friendly, affordable and accurate digital particle image velocimetry in MATLAB, J. Open Res. Softw. 2 (2014) 1-10.

[30] S.M. Pizer, E.P. Amburn, J.D. Austin, R. Cromartie, A. Geselowitz, T. Greer, B. ter H. Romeny, J.B. Zimmerman, K. Zuiderveld, Adaptive histogram equilization and its variations, Comput. Vis. Graph Image Process. 39 (1987) 355-368.

[31] Z. Carr, K. Ahmed, D. Forliti, Spatially correlated precision error in digital particle image velocimetry measurements of turbulent flows, Exp. Fluids 47 (2009) 95-106.

[32] S. Pfadler, F. Beyrau, A. Liepertz, Flame front detection and characterization using conditioned particle image velocimetry (CPIV), Opt. Express 15 (2007) 15444-15456.

[33] S. Pfadler, F. Dinkelacker, F. Beyrau, A. Liepertz, High resolution dual-plane stereo-PIV for validation of subgrid scale models in large-eddy simulations of turbulent premixed flames, Combust. Flame. 156 (2009) 1552-1564.

[34] A.M. Steinberg, J.F. Dirscoll, Straining and wrinkling processes during turbulencepremixed flame interaction measured using temporally-resolved diagnostics, Combust. Flame 156 (2009) 2285-2306.

[35] G.A. Moore, Automatic scanning and computer processes for the quantative analysis of micrographs and equivalent subjects, Pict. Pattern Recognit. 31 (1968) 275-362.

[36] N. Otsu, A threshold selection method from gray-level histograms, IEEE Trans. Syst. Man. Cybern. 9 (1979) 62-66.

[37] C. Tang, Z. Huang, C. Jin, K. He, X. Wang, H. Miao, Laminar burning velocities and combustion characteristics of propane-hydrogen-air premixed flames, Int. J. Hydrogen Energy 33 (2008) 4906-4914.

[38] C.M. Vagelopoulos, F.N. Egolfopouols, C.K. Law, Further considerations on the determination of laminar flame speeds with the counterflow twin-flame technique, Proc. 
Combust. Inst. 25 (1994) 1341-1347.

[39] S. Chaudhuri, S. Kostka, S.G. Tuttle, M.W. Renfro, B.M. Cetegen, Blowoff mechanism of two dimensional bluff-body stabilized turbulent premixed flames in a prototypical combustor, Combust. Flame 158 (2011) 1358-1371.

[40] A.E. Lutz, R.J. Kee, J.F. Grcar, F.M. Rupley, OPDIFF: A fortran program for computing opposed-flow diffusion flames, Livermore, CA, USA, 1997. Report No. SAND96-8243.

[41] M.K. Geikie, K.A. Ahmed, Lagrangian mechanisms of flame extinction for lean turbulent premixed flames, Fuel 194 (2017) 239-256.

[42] A.M. Steinberg, B. Coriton, J.H. Frank, Influence of Combustion on principal strain-rate transport in turbulent premixed flames, Proc. Combust. Inst. 35 (2015) 1287-1294.

[43] H. Weller, G. Tabor, A. Gosman, C. Fureby, Application of a flame-wrinkling les combustion model to a turbulent mixing layer, Symp. (Int.) Combust. 27 (1998) 899-907.

[44] P. Welch, The use of fast fourier transform for the estimation of power spectra: A method based on time averaging over short, modifed periodograms, IEEE Trans. Audio Electroacoust. 15 (1967) 70-73.

[45] A. Prasad, C.H.K. Williamson, The instability of the shear layer seperating from a bluff body, J. Fluid Mech. 333 (1997) 375-402.

[46] J.C. Pan, M.D. Vangsness, D.R. Ballal, Aerodynamics of bluff-body stabilized confined turbulent premixed flames, J. Eng. Gas Turbines Power 114 (1992) 783-789. 